\title{
CMOS driven micro-pixel LEDs integrated with single photon avalanche diodes for time resolved fluorescence measurements
}

\author{
B R Rae ${ }^{1,4}$, C Griffin ${ }^{2}$, J McKendry ${ }^{2}$, J M Girkin ${ }^{2}$, H X Zhang Z E Gu $^{2}$, \\ D Renshaw ${ }^{1}$, E Charbon ${ }^{3}$, M D Dawson ${ }^{2}$ and R K Henderson ${ }^{1}$ \\ ${ }^{1}$ Institute for Integrated Micro and Nano Systems, The School of Electrical and Electronic Engineering, \\ The University of Edinburgh, The King's Buildings, Mayfield Road, Edinburgh, EH9 3JL, UK \\ 2 Institute of Photonics, SUPA, University of Strathclyde, 106 Rottenrow, Glasgow G4 0NW, UK \\ ${ }^{3}$ Ecole Polytechnique Fédérale de Lausanne (EPFL), CH-1015, Lausanne, Switzerland \\ E-mail: Bruce.Rae@ed.ac.uk
}

Received 17 September 2007, in final form 6 December 2007

Published 4 April 2008

Online at stacks.iop.org/JPhysD/41/094011

\begin{abstract}
We describe a single chip approach to time resolved fluorescence measurements based on time correlated single photon counting. Using a single complementary metal oxide silicon (CMOS) chip, bump bonded to a $4 \times 16$ array of AlInGaN UV micro-pixellated light-emitting diodes, a prototype integrated microsystem has been built that demonstrates fluorescence excitation and detection on a nanosecond time scale. Demonstrator on-chip measurements of lifetimes of fluorescence colloidal quantum dot samples are presented.
\end{abstract}

(Some figures in this article are in colour only in the electronic version)

\section{Introduction}

The use of fluorescence-based methodologies is at the core of many modern instrumentation technologies, especially in the life sciences. Originally, the interest was in the specific labelling of biological samples for imaging applications, but more recently this has expanded rapidly with the growth of DNA sequencing and micro-array applications. In principle, the equipment needed for such spectroscopic instrumentation has not changed over several decades, in that a narrow wavelength source is required to excite the fluorophore of interest and the resulting fluorescence must pass through an optical device to separate the excitation light from the fluorescence emission, before being detected by a light sensitive instrument and the level of fluorescence determined. However, using modern fabrication and manufacturing techniques, such instrumentation can now be made in complete integrated systems with the potential for volume production. The range of methods for interpreting the fluorescence data has

\footnotetext{
${ }^{4}$ Author to whom any correspondence should be addressed.
}

also expanded with the growth of fast electronics, originally destined for the telecommunications and computer markets, now being applied to fluorescence instrumentation.

Most commonly used fluorophores are designed to be excited in the near ultraviolet (UV) and blue regions of the optical spectrum. The advent of GaN and other blue emitting semiconductor sources, around 15 years ago [1], has helped stimulate interest in the use of such sources in place of lasers or mercury discharge lamps for excitation in fluorescence-based instrumentation. The advantages of these sources include low cost, reliability, compactness and good wavelength matching to many standard fluorophores. In addition, their ability to produce short $(\approx 1 \mathrm{~ns})$ pulse lengths offers the opportunity for low cost fluorescence lifetime measurements. It has been appreciated for a long time [2] that the fluorescence decay from a molecule provides a significant level of information about the sample and the surrounding conditions of the fluorophore, from the local viscosity in cell membranes [3] to $\mathrm{pH}$ [4]. Most recently interest has been in the rapid growth of Föster resonance energy transfer (FRET) methods, where the spatial separation of two fluorescent molecules significantly affects 
the fluorescence lifetime of the shorter wavelength 'donor' molecule. Through advances in molecular chemistry, FRET based assays and experiments utilizing lifetime measurement as the monitor of the FRET process are emerging as the method of choice for many applications in the life sciences [5].

Several methods of lifetime measurement are now in common use in lifetime instrumentation, but all require the common features of a short pulsed, or high frequency modulated, light source, fast detector and timing electronics. Time correlated single photon counting (TCSPC) is potentially the most accurate method of determining complex decays (multiple lifetimes) and was therefore the method selected to demonstrate the performance of our system. The phase method, in which the phase change in the fluorescence emission relative to the excitation pulse is measured [6], is less sensitive compared with TCSPC and applies more light to the sample which can lead to faster sample decomposition. A slight variation on TCSPC is the use of time gated detectors which provide a slightly less accurate lifetime measurement, but are excellent at providing a method of detecting changes in lifetime [7]. More recently, a variation on the phase method has been developed, whereby the change in output intensity is measured [8]. This method uses a slow detector and thus was not suitable in a system that makes use of rapid detectors. Therefore, we chose the TCSPC method as we believed that this would demonstrate the capability of our novel excitation source and detector. Furthermore, if the system worked in this configuration it should also work in the slightly less demanding phase and gated configurations, though these might be the preferred options in a final instrument configuration.

In 1995, Araki and Misawa [9] and in 2005, Davitt et al [10] both reported the use of light-emitting diodes (LEDs) for lifetime measurements; however, their system used a conventional photomultiplier. Although this work demonstrated the capability of LEDs for lifetime measurements with the use of a photomultiplier as the detector, the system was not capable of low cost miniaturization. In this paper, we describe a single chip embodiment for such measurements based on time correlated single photon counting. In our approach, a $16 \times 4$ array of micropixel AlInGaN LEDs emitting at $370 \mathrm{~nm} \mathrm{[11]} \mathrm{has} \mathrm{been}$ bonded to an equivalent array of aluminium electrodes made from the top metal of a $0.35 \mu \mathrm{m}$ high-voltage CMOS chip. Each electrode has an associated driver circuit capable of switching up to $50 \mathrm{~V}$. Incorporated into each electrode is a single photon avalanche diode (SPAD). The output of the SPAD detectors is processed in real time by a TCSPC module that generates a histogram of the fluorescence decay [12]. Other pertinent features of the CMOS chip include on-chip control of the micro-LED pulse width (1.5-48 ns optical pulse width) and the ability to separately address any micro-LED or SPAD in the array. With this device, we have successfully performed demonstration measurements in a colloidal suspension of quantum dots, achieving lifetimes consistent with manufacturer's specifications and our own reference measurements taken using a photomultiplier tube detector. This microsystem solution will enable the development of low cost, portable fluorescence lifetime readers

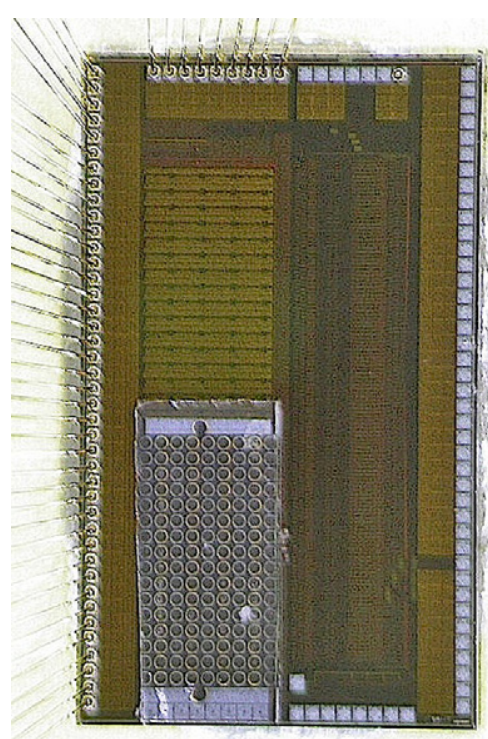

Figure 1. Micrograph image of CMOS chip with bump-bonded micro-LED array.

for many optical lab-on-a-chip applications such as point-ofcare diagnostics equipment and the synthesis and/or read out of DNA micro-arrays. With each element being separately addressable, there is the potential to excite many fluorescent samples in parallel [11]. By integrating the excitation source with a photodetector and on-chip driving electronics, our devices will contribute to the development of lab-on-a-chip (LoC) systems [13]. This includes work by Chodavarapu et al [14], which aims to carry out fluorescence detection using a CMOS-based system which incorporates a detector with signal processing circuitry. Their system does not, however, include an integrated excitation source and uses a discrete LED. Cleary et al [15] demonstrated TCSPC on a microscale using integrated optics and microfluidics; however, their system relies on a pulsed diode laser as an excitation source and a discrete SPAD detector. The system presented here offers a greater level of integration, placing the excitation source in the same micro-scale system as the sample and detector.

\section{Experimental setup}

A micrograph image of the CMOS chip with bump-bonded micro-LED array can be seen in figure 1 .

The micro-LEDs consist of a $16 \times 4$ array of individually addressable pixels, each pixel having a diameter of $72 \mu \mathrm{m}$ on a $100 \mu \mathrm{m}$ pitch. The devices were fabricated from 'standard' UV LED wafers grown on $c$-plane sapphire substrates by metal organic chemical vapour deposition and have a peak emission at $370 \mathrm{~nm}$ [16]. The array of electrodes was depassivated by pad opening at the foundry to reveal the aluminium topplate electrodes for bump bonding. A post-processing step of oxygen plasma etching removed the polyimide layer of the chip which has been shown to improve the photon detection probability (PDP) of the underlying SPADs by a factor of 2-5 [17]. An example of the top metal driver plates can be seen in figure 2. An electrical connection between the micro-LED 
array and the driving CMOS chip was made using a bumpbonding process with each element of the micro-LED array being bonded to a dedicated driver site. A four metal layer, high voltage, $0.35 \mu \mathrm{m}$ CMOS technology was used to fabricate the driving CMOS chip. The first two layers of metal were used for signal routing, the third layer formed a shield to protect the underlying electronics from the high-voltage signal being driven onto the array and the fourth layer was used to pattern an array of driver plates onto which the micro-LED array was bonded. Driving circuitry for each electrode and SPAD is contained within a $200 \times 100 \mu \mathrm{m}^{2}$ cell, with $19090 \mu \mathrm{m}^{2}$ of top metal exposed as the electrode surface. Electrode selection is controlled using row and column addressing.

The SPAD used in this test chip is essentially an avalanche photodiode operating in Geiger mode. This mode of operation enables detection of single photons, when the diode is equipped with the means to detect and regenerate the avalanche currents associated with photon detection. A schematic cross section of a SPAD is shown in figure 3. The high voltage, twin-tub CMOS technology allows the construction of a $\mathrm{p}+$ /deep $n$-tub avalanche multiplication region. Premature lateral breakdown of the junction is avoided by a guard ring of lightly doped p-type diffusion. A detailed description of the SPAD structure

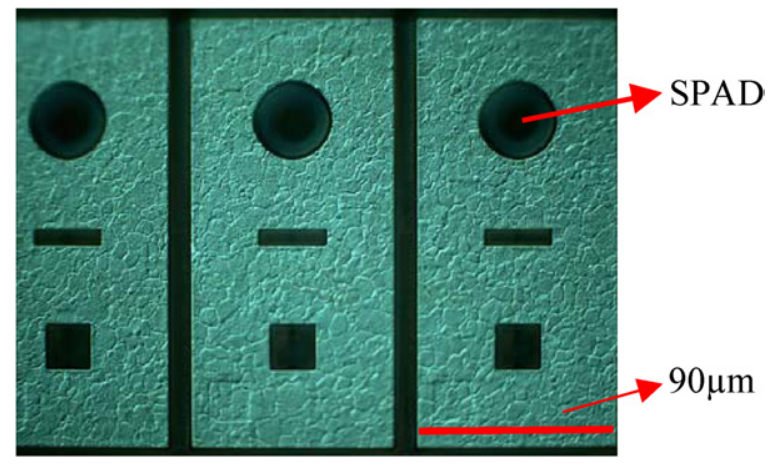

Figure 2. Scanning electron microscope (SEM) image of the individual top layer metal plates upon which each micro-LED in the array is bump bonded. The SPAD detectors are situated in the circular gap in the metal. and measured performance can be found in [18]. The detection of a photon and the subsequent avalanche breakdown of the SPAD generates a voltage pulse, the duration of which is approximately $30 \mathrm{~ns}$, which is quenched passively on chip. Single incident photons generate digital pulses, with around 100 ps of timing jitter, which are readily processed by on-chip electronics.

Measurement of fluorescence decay curves have been demonstrated using $\mathrm{CdSe} / \mathrm{ZnS}$ quantum dots (Evident Technologies Inc., USA) in a toluene solution [19] at concentrations as they were received from the supplier. These particles have an excitation spectrum that peaks at wavelengths less than $400 \mathrm{~nm}$ and emissions at $526 \mathrm{~nm}$ (Adirondack Green), $543 \mathrm{~nm}$ (Catskill Green) and $555 \mathrm{~nm}$ (Hops Yellow), respectively. Table 1 contains a summary of the quantum dot samples used.

The samples are held in a cavity microslide (Agar Scientific Ltd, UK) and sealed by a $24 \mathrm{~mm} \times 24 \mathrm{~mm}$ cover slip to avoid evaporation. The slide is placed on top of the chip and excited by a pulse train from a single micro-LED. The LED is biased with $4 \mathrm{~mA}$ of bias current and the electrode driven to $13 \mathrm{~V}$, producing short pulses with fast rise and fall times ( $2.15 \mathrm{~ns}$ and $3.31 \mathrm{~ns}$ respectively, for a pulse width of $8 \mathrm{~ns}$ ). Each micro-LED driver site also contains an addressable SPAD detector, as described above. The sapphire substrate of the micro-LED array is optically transparent and therefore any returning fluorescence light passes through the micro-LED array and reaches the SPAD array. Figure 4 shows a schematic of the optical stack.

The number and position of the LED pixels that are active is user definable and any combination from a single LED up to all 64 elements of the array can be active at any one time. During analysis all 64 SPADs in the array are active. However, the address chosen by the user defines which SPADs are present on the output bus; thus, the address of the detecting SPAD does not have to correspond to the address of the exciting micro-LED. To avoid direct coupling of excitation light from the micro-LED, the SPAD chosen to detect is situated eight rows away $(800 \mu \mathrm{m})$ from the emitting LED pixel. It should be noted that this does not completely

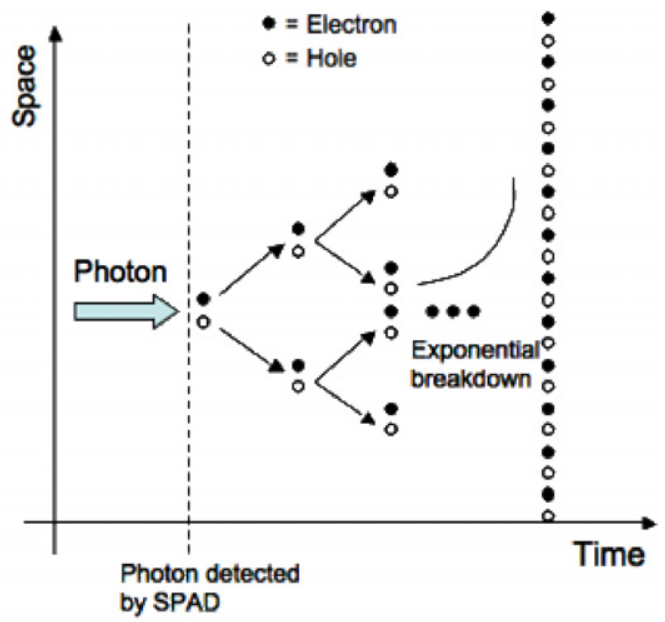

Figure 3. Schematic cross section of CMOS SPAD and illustration of avalanche breakdown. 
Table 1. Summary of quantum dot samples used to evaluate system.

\begin{tabular}{lllll}
\hline Sample & $\begin{array}{l}\text { Peak } \\
\text { absorption } \\
(\mathrm{nm})\end{array}$ & $\begin{array}{l}\text { First } \\
\text { absorption } \\
\text { peak }(\mathrm{nm})\end{array}$ & $\begin{array}{l}\text { Emission } \\
\text { peak }(\mathrm{nm})\end{array}$ & $\begin{array}{l}\text { Concentration } \\
\left(\mathrm{nmol} \mathrm{ml}^{-1}\right)\end{array}$ \\
\hline $\begin{array}{c}\text { Adirondack } \\
\text { Green }\end{array}$ & $<400$ & 510 & 526 & 77.03 \\
$\begin{array}{c}\text { Catskill } \\
\text { Green }\end{array}$ & $<400$ & 525 & 543 & 68.77 \\
$\begin{array}{c}\text { Hops } \\
\text { Yellow }\end{array}$ & $<400$ & 531 & 555 & 57.17 \\
\hline
\end{tabular}

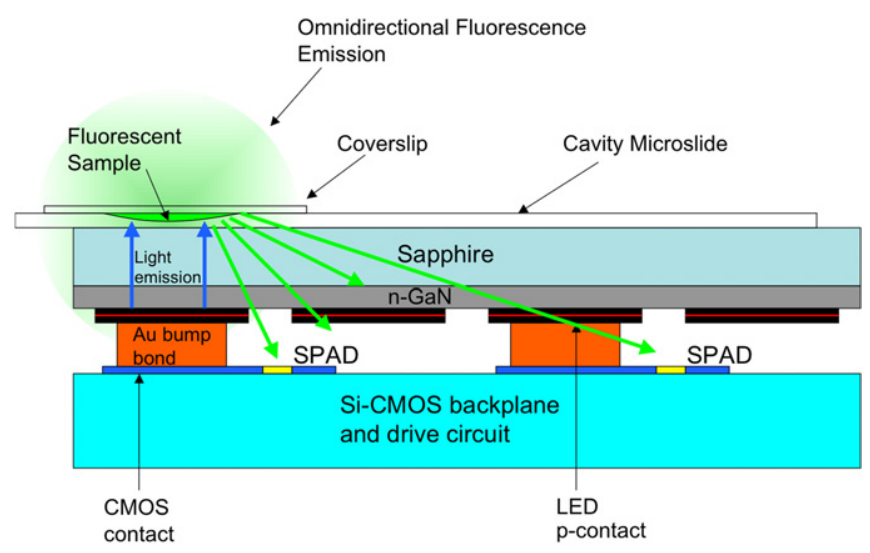

Figure 4. Schematic overview of experimental setup. The transparent sapphire substrate allows the fluorescence emission to pass and reach the SPAD below.

eliminate photon counts due to direct LED excitation of the SPAD. Photon counts due to directly coupled light from the LEDs have been further minimized by choosing a $370 \mathrm{~nm}$ UV excitation source. At $370 \mathrm{~nm}$, the PDP of the SPADs is $7 \%$. However, this wavelength is ideal for the excitation of the selected quantum dots. At $526 \mathrm{~nm}$ (the emission wavelength of the Adirondack Green quantum dots) the PDP of the SPADs increases to $25 \%$ (after depassivation). This insensitivity to shorter wavelengths results in filtering of the excitation as summarized in figure 5, which shows the spectral characteristics of the LED, Adirondack Green sample and SPAD. The LED and Adirondack Green emission spectra were measured using a Triax 550 spectrometer, with the Adirondack Green excited by a Power Technology $15 \mathrm{~mW}$, $375 \mathrm{~nm}$ UV laser. For the absorption, $200 \mu \mathrm{l}$ of Adirondack Green was mixed with $1800 \mu$ l of toluene in a quartz cuvette and its absorption spectrum measured with a Lambda 2 spectrophotometer.

Each micro-LED pulse is synchronized to the rising edge of the system clock. This clock is therefore used as the synchronization pulse for the TCSPC card (Becker and Hickl, SPC-730). The TCSPC card is operated in reverse mode and the pulse train from the SPADs are passed via a pulse inverter to the constant fraction discriminator input of the card. The micro-LED pulse frequency is set by the system clock and is user definable, for these experiments a pulse frequency of $3.6864 \mathrm{MHz}$ was chosen. The micro-LED electrical drive pulse width is also user definable via software control of the chip. The user can select pulse widths from $400 \mathrm{ps}$ up to $48 \mathrm{~ns}$ in steps of $400 \mathrm{ps}$. This allows the user to select an excitation pulse that best suits the sample of interest. For the quantum dots used in this study, an excitation pulse width of $8 \mathrm{~ns}$ was chosen. A photograph of the micro-LED array exciting a sample of Adirondack Green quantum dots mounted on a micro-cavity slide is shown in figure 6 .

\section{Results}

The signal generated by the on-chip SPADs is passed to the TCSPC card which creates a histogram based on photon arrival times, relative to the excitation pulse. As mentioned above, due to the absence of any optical spectral filtering in the system, the excitation pulse is also detected by the SPADs and can be observed in the resulting histogram as the large broad peak on the left. The ratio of detected excitation light to detected fluorescence emission is dependent on several factors. One such factor is the intensity of excitation light from the LED. This can be controlled by the user by adjusting the peak voltage, the bias current of the driver and the width of the excitation pulse. The intensity of fluorescence emission from the sample is also an important factor. Finally, the relative distance from the excitation micro-LED and the relevant detection SPAD affects the level of directly coupled light from the LED reaching the detector. This affects the ratio of the number of counts due to the LED pulse and the fluorescence emission, as demonstrated in figure 7.

As can be seen from the results in figure 7, the fluorescence decay curves can be clearly distinguished from the instrument response function (IRF). The data gathered are exported and analysed using FAST $^{\mathrm{TM}}$ lifetime extraction software by Edinburgh Instruments. Discrete deconvolution of the IRF and decay curves presented in figure 7 yields lifetimes of $17.8 \mathrm{~ns}$, $17.2 \mathrm{~ns}$ and $19.1 \mathrm{~ns}( \pm 104 \mathrm{ps}$ estimated measurement error, based on $100 \mathrm{ps}$ jitter from the SPAD and 4 ps resolution of the TCSPC hardware) for Adirondack Green, Catskill Green and Hops Yellow, respectively. This is in good agreement with the lifetimes quoted by the manufacturer (15-20 ns) and measured independently using a photomultiplier tube as a detector. The noise floor of the results presented in figure 7 is also in good agreement with the $50 \mathrm{~Hz}$ dark count rate (DCR) of the SPADs quoted in the literature, which is strongly temperature dependent [17].

\section{Conclusions and discussion}

A compact fluorescence lifetime analysis system has been demonstrated, which incorporates sample excitation via a CMOS driven UV $16 \times 4$ micro-LED array and emission detection using an array of SPAD detectors located on the same CMOS chip. The AlInGaN micro-LED array was bump bonded to the SPAD and driver array which has been fabricated in a high-voltage $0.35 \mu \mathrm{m}$ CMOS technology and allows the user to define the excitation pulse and intensity.

The CMOS micro-LED driver was designed to deliver electrical pulses of variable width, from $400 \mathrm{ps}$ to $48 \mathrm{~ns}$. As a result of a larger than expected load capacitance, due to the bump-bonded micro-LED elements an optical pulse of 400 ps was not achieved. Figure 8 demonstrates the longest 


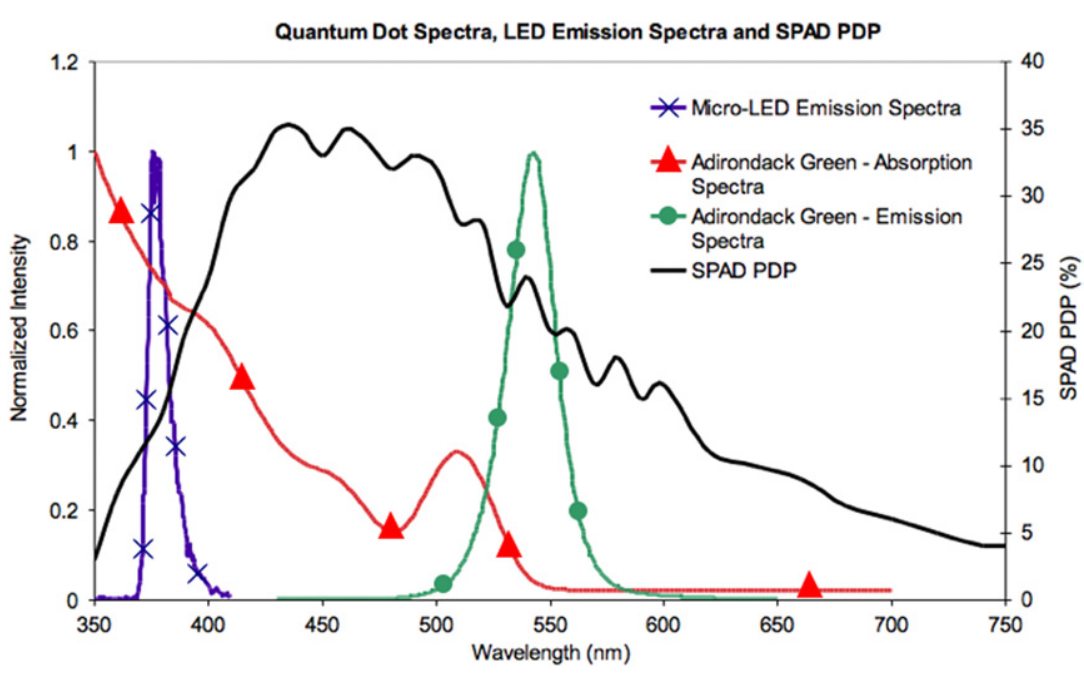

Figure 5. Graph of micro-LED emission spectra, SPAD PDP and quantum dot absorption (Adirondack Green) and emission spectra versus wavelength. This clearly highlights the spectral separation of the excitation wavelength and the detector sensitivity range.

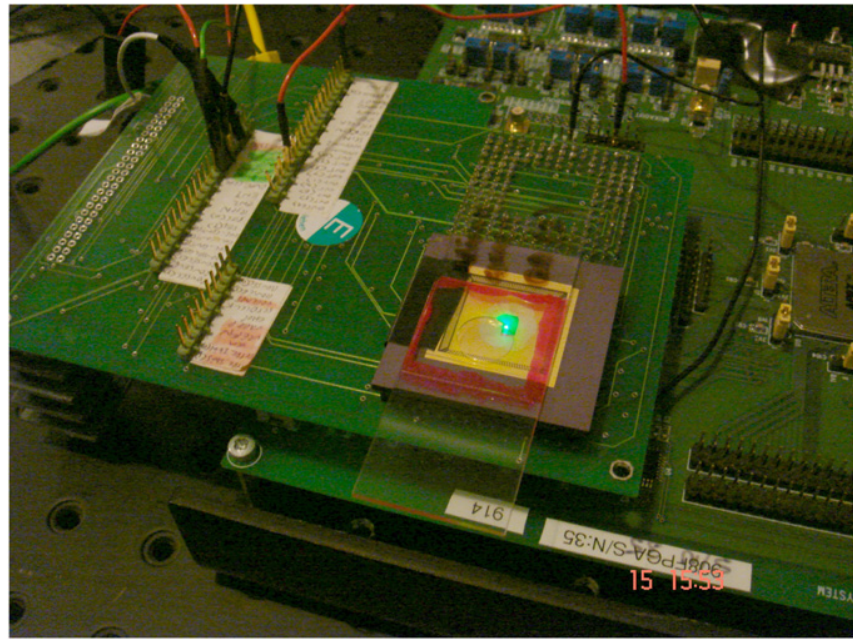

Figure 6. Photograph of micro-LED array exciting a sample of Adirondack Green quantum dots on a micro-cavity slide.

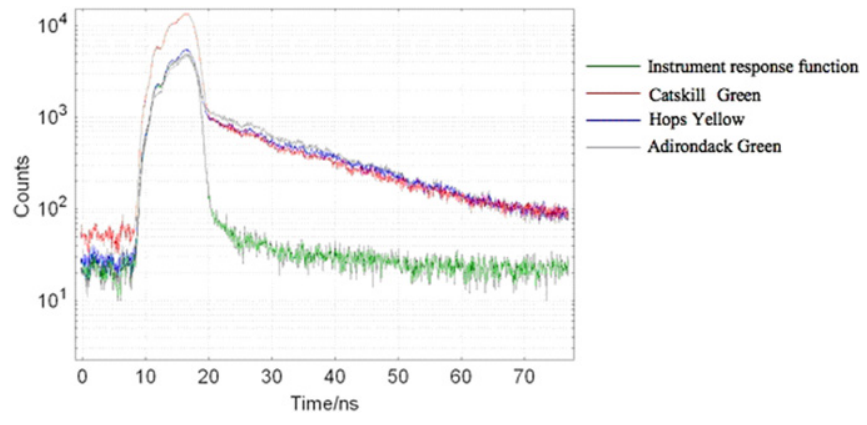

Figure 7. IRF and fluorescent decay curves for quantum dots at three different emission wavelengths.

(47.87 ns full width half maximum, FWHM) and shortest (1.12 ns, FWHM) optical pulses achieved. While it has been shown that a pulse of $1.12 \mathrm{~ns}$ (FWHM) is possible, a pulse of $8 \mathrm{~ns}$ was chosen to conduct the lifetime experiments. As the pulse width is decreased, the intensity of the micro-LED emission is reduced, which in turn reduces the fluorescence

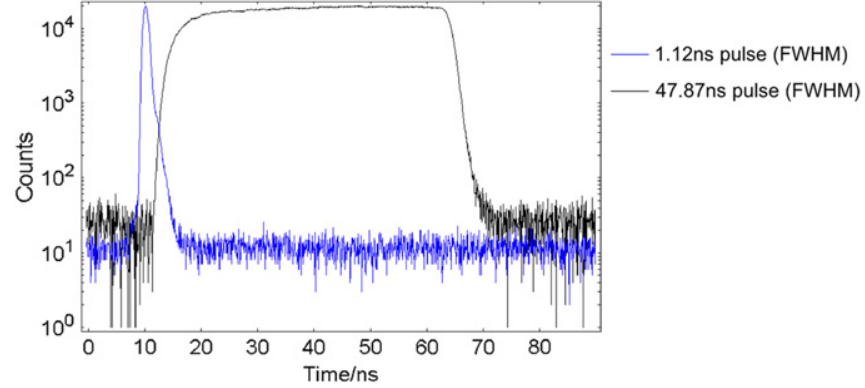

Figure 8. The longest ( $47.87 \mathrm{~ns}, \mathrm{FWHM})$ and shortest (1.12 ns, FWHM) optical pulses achieved. Greater than expected capacitive load on the driver circuit output prevented shorter pulses being achieved.

emission. This leads to a lower signal-to-noise ratio and thus a less accurate lifetime measurement. To allow an $8 \mathrm{~ns}$ excitation pulse to be used, fluorophores with a relatively long lifetime were used. In addition to their compatible wavelength properties, as described previously, the 15-20 ns lifetimes of the quantum dot samples were ideally matched for excitation with an $8 \mathrm{~ns}$ excitation pulse.

Results have been presented which demonstrate the system's ability, in conjunction with external TCSPC hardware, to accurately measure fluorescence lifetime data of commercially available quantum dot fluorophore samples. The system removes the need for complicated optical alignment, with the sample being introduced into the system on a simple micro-cavity slide. The SPADs natural insensitivity to UV wavelengths has also been used to remove the need for optical filters. This system is, to the best of our knowledge, the first demonstration of a complete microsystem for TCSPC to incorporate both an array of excitation sources and an array of detectors with single photon sensitivity. While results are not yet comparable to more established experimental setups using commercially available equipment, this prototype system demonstrates the benefits and potential of an integrated system. The ability of such a microsystem, based on a standard CMOS process, to conduct lifetime analysis without 
the need for expensive optics and filters should open the way to the development of a compact (potentially portable), robust and inexpensive solution to time resolved fluorescence measurements.

\section{Acknowledgments}

This work was funded by the UK Engineering and Physical Sciences Research Council, in part under the Basic Technology programme and under a Scottish Funding Council SRDG Award. We are grateful to Optocap Ltd, Livingston, UK for carrying out the bump-bonding process. We further thank Alec Ruthven at the Scottish Microelectronic Centre, Edinburgh, for chip bonding, the COSMIC laboratory facility at the University of Edinburgh for providing access to test equipment and laboratory space, Dr Paul R Edwards and Professor Robert W Martin at the Department of Physics, University of Strathclyde, for providing the quantum dot samples and Dr Olaf J Rolinski and Professor David J S Birch for allowing access to the spectrophotometer for absorption measurements.

\section{References}

[1] Nakamura S and Fasol G 1997 The Blue Laser Diode: GaN Based Light Emitters and Lasers (New York: Springer)

[2] Lakowicz J R 1999 Principles of Fluorescence Spectroscopy 2nd edn (Dordrecht/New York: Kluwer/Plenum)

[3] Kung C E and Reed J K 1986 Microviscosity measurements in phospholipids bilaters using fluorescent dyes that undergo torsional relaxation Biochemistry 32 6114-21

[4] Sipior J, Carterm G M, Lakowicz J R and Rao G 1997 Blue light emitting diode demonstration as an ultra-violet excitation source for nanosecond phase-modulation fluorescence lifetime measurements Rev. Sci. Instrum. $682666-70$

[5] Jares-Erijman E A and Jovin T M 2003 FRET Imaging Nature Biotechnology 21 1387-95

[6] Gratton E and Limkeman M 1983 A continuosly variable frequency cross-correlation phase fluorometer with picosecond resolution Biophys. J. 44 215-324

[7] Buurman E P, Sanders R, Draaijer A, Gerritsen H C, Van Veen J J, Houp P M and Levine Y K 1992 Fluorescence lifetime imaging using a confocal laser scanning microscope Scanning 14 155-59

[8] Matthews D R, Summers H D, Njoh K, Errington R J, Smith P J, Barber P, Ameer-Beg S and Vojnovic B 2006 Technique for measurement of fluorescence lifetime by use of stroboscopic excitation and continuous-wave detection Appl. Opt. 45 2115-23

[9] Araki T and Misawa H 1995 Light emitting diode-based nanosecond ultraviolet light source for fluorescence lifetime measurements Rev. Sci. Instrum. 66 5469-72

[10] Davitt K, Song Y-K, Patterson W, Nurmikko A, Gherasimova M, Han J, Pan Y-L and Chang R 2005290 and $340 \mathrm{~nm}$ UV LED arrays for fluorescence detection from single airborne particles Opt. Express 13 9548-55

[11] Griffin C, Gu E, Choi H, Jeon C, Rolinski O, Birch D, Girkin J and Dawson M 2004 Fluorescence excitation and lifetime measurements using Ga/InGaN micro-LED arrays The 17th Annual Meeting of the IEEE Lasers and Electro-Optics Society (Western Rio Mar, Puerto Rico) vol 2 pp 896-97

[12] Becker W 2005 Advanced Time-correlated Single Photon Counting Techniques (Springer Series in Chemical Physics) (Berlin: Springer)

[13] Voh-Dinh T and Askari M 2001 Micro-arrays and biochips: applications and potential in genomics and proteomics J. Curr. Genomics 2 399-415

[14] Chodavarapu V P, Shubin D O, Barkowski R M, Titus A H, Cartwright A N and Bright F V 2007 CMOS-based phase fluorometric oxygen sensor system IEEE Trans. Circuits Syst. 54 111-18

[15] Cleary A, Glidle A, Laybourn P J R, Garcia-Blanco S, Pellegrini S, Helfter C, Buller G S, Aitchison J S and Cooper J M 2007 Integrating optics and microfluidics for time-correlated single-photon counting in lab-on-a-chip devices Appl. Phys. Lett. 91071123

[16] Gong Z, Zhang H X, Gu E, Griffin C, Dawson M D, Poher V, Kennedy G, French P M W and Neil M A A 2007 Matrix-addressable micropixellated InGaN light-emitting diodes with uniform emission and increased light output IEEE Trans. Electron. Devices 54 2650-8

[17] Niclass C, Sergio M and Charbon E 2006 A single photon avalanche diode array fabricated in deep-submicron CMOS technology Proc. Conf. on Design, Automation and Test in Europe (Munich, Germany) pp 81-6

[18] Niclass C, Rochas A, Besse P-A, Popovic R S and Charbon E 2005 CMOS imager based on single photon avalanche diodes IEEE Transducers 1 1030-4

[19] Evident Technologies EviDot Specifications http://www. evidenttech.com/products/evidots/evidot-specifications.html 